Wilfrid Laurier University

Scholars Commons @ Laurier

Kinesiology and Physical Education Faculty

Publications

Kinesiology and Physical Education

2010

\title{
The Role of Families in Youth Sport Programming in a Canadian Aboriginal Reserve
}

\author{
Robert Schinke \\ Laurentian University \\ Hope Yungblut \\ Laurentian University \\ Amy Blodgett \\ Laurentian University \\ Mark A. Eys \\ Wilfrid Laurier University, meys@wlu.ca \\ Duke Peltier \\ Wikwemikong Sport and Recreation Program
}

See next page for additional authors

Follow this and additional works at: https://scholars.wlu.ca/kppe_faculty

\section{Recommended Citation}

Schinke, Robert; Yungblut, Hope; Blodgett, Amy; Eys, Mark A.; Peltier, Duke; Ritchie, Stephen; and RecolletSaikkonen, Danielle, "The Role of Families in Youth Sport Programming in a Canadian Aboriginal Reserve" (2010). Kinesiology and Physical Education Faculty Publications. 19.

https://scholars.wlu.ca/kppe_faculty/19

This Article is brought to you for free and open access by the Kinesiology and Physical Education at Scholars Commons @ Laurier. It has been accepted for inclusion in Kinesiology and Physical Education Faculty Publications by an authorized administrator of Scholars Commons @ Laurier. For more information, please contact scholarscommons@wlu.ca. 


\section{Authors}

Robert Schinke, Hope Yungblut, Amy Blodgett, Mark A. Eys, Duke Peltier, Stephen Ritchie, and Danielle Recollet-Saikkonen 


\title{
The Role of Families in Youth Sport Programming in a Canadian Aboriginal Reserve
}

\author{
Robert Schinke, Hope Yungblut, Amy Blodgett, Mark Eys, Duke Peltier, \\ Stephen Ritchie, and Danielle Recollet-Saikkonen
}

\begin{abstract}
Background: There has been a recent push in the sport psychology literature for sport participants to be approached based on their cultural backgrounds. However, there are few examples where a cultural approach is considered, such as a culturally reflexive version of participatory action research (PAR). In the current study, the role of family is considered in relation to the sport engagement of Canadian Aboriginal youth. Methods: Mainstream researchers teamed with coresearchers from the Wikwemikong Unceded Indian Reserve for 5 years. Community meetings and talking circles were employed as culturally sensitive data collection techniques to uncover how to encourage youth participation in Wikwemikong's sport programs. The overarching methodology for the project is PAR. Results: Themes and subthemes were determined by community consensus with terms indigenous (ie, culturally relevant) among the local Aboriginal culture. Family was considered important for youth involvement in Aboriginal community sport programs. Parents were expected to support their children by managing schedules and priorities, providing transportation, financial support, encouragement, and being committed to the child's activity. Aunts, uncles, cousins, siblings, grandparents, and the family as a whole were seen as sharing the responsibility to retain youth in sport through collateral support (ie, when gaps in parental support arose). Conclusions: Suggestions are proposed regarding how families in Aboriginal communities can collaborate to facilitate sport and physical activity among their youth. Further suggestions are proposed for researchers engaging in culturally reflexive research with participants and coresearchers from oppressed cultures.
\end{abstract}

Keywords: physical activity, community programming, qualitative research

Recently there has been a push in sport psychology for athletes to be approached based on their cultural backgrounds. ${ }^{1,2}$ The term for culturally reflexive sport psychology is cultural sport psychology (CSP). The intent through CSP is to foreground relevant approaches to sport psychology within each culture, as opposed to continuing with 1 monolithic approach. ${ }^{1}$ To do otherwise is to risk silencing the views of sport participants from minority cultures to the point where those who are supposed to be understood through effective research and in applied service are asked to reposition their perspective and adopt the mainstream norms within the region. ${ }^{2}$ Consequently, given the vast cultural backgrounds that sport participants come from, some have been relocated to the margins through oppression. Despite a predominantly mainstream sport psychology, meaning a monocultural approach taken with athletes regardless of their culture, authors have considered nuances among (a) peoples of

Schinke, Yungblut, Blodgett, Eys, and Ritchie are with the School of Human Kinetics, Laurentian University, Sudbury, Ontario, Canada. Peltier and Recollet-Saikkonen are with the Wikwemikong Sport and Recreation Program, Wikwemikong Unceded Indian Reserve, Wikwemikong, Ontario, Canada. color (eg, Latin, African American, Arabic) in the United States, ${ }^{3,4}$ (b) Australian Aborigines, ${ }^{5}$ and relevant herein, (c) Canadian Aboriginal athletes. ${ }^{6,7}$ Consistent among the contributions is a call for culturally reflexive approaches to our work as sport scientists and physical educators, developed with (and not for) the intended population.

Herein, the authors from 2 cultures have collaborated as a bicultural research team, where the focus has gradually shifted toward youth sport participation, and away from elite athletes. ${ }^{8}$ The transition has been at the suggestion of the Canadian Aboriginal coauthors and their Aboriginal community: Wikwemikong Unceded Indian Reserve. Wikwemikong is an unceded Indian Reserve in Northern Ontario, Canada. It is located on Manitoulin Island, approximately 150 miles from Laurentian University, where the academic researchers work. The closest major city to Wikwemikong is Toronto, Canada, 350 miles and approximately 7 hours driving distance away. Wikwemikong's residents have been challenged by low levels of sport participation in their youth sport programs despite these programs being the community's primary sport and physical activity opportunity for the cohort. ${ }^{8}$ As Blodgett and colleagues learned, earlier community attempts at sport programming in Wikwemikong (ie, before the research team began to collaborate) resulted 
in limited volunteer resources, sparsely attended sport and activity programs, and a frustration among sport staff regarding how to enhance their services. Contextually, what Wikwemikong experiences is in keeping with a national trend pertaining to sedentary behavior and health risks generally among youth residing in Aboriginal Reserves. From Canada's national statistics, it has been indicated that Aboriginal youth suffer above average in terms of Type One diabetes, ${ }^{9}$ substance abuse, ${ }^{10,11}$ obesity, heart problems, and they have a shorter life expectancy. ${ }^{12}$ Though health records are sparse in Wikwemikong, it is well known among the members of Band and Council that Wikwemikong youth are both an at risk and also a vibrant cohort within the community. Pertaining to sport and activity, it has been noted by sport and recreation staff as well as staff from the Wikwemikong youth center that youth engage in sport and physical activity until they begin grade 9, after which enrollment in physical activity programs drops rapidly (ie, many programs are offered without any attendees). Consequently, the intent has been to develop sport programs with the intended community, seeking feedback from Wikwemikong stakeholders. The belief is that through informed programs derived from within the intended community, the Wikwemikong members could develop practices to counter health challenges in the community through sport and physical activity.

Focus is placed here on family members, and what role they play in the sport engagement of Aboriginal community youth. Further, the approach to the research is Participatory Action Research (PAR), with aspects that have originated from within Wikwemikong. As Smith has articulated in relation to methodologies that empower indigenous peoples, "Real power lies in those who design the tools. ... Contained within this imperative is a sense of being able to determine priorities, to bring to the centre those issues of our own choosing, and to discuss them amongst ourselves" (p. 38). ${ }^{13}$ What follows pertains to the role of family in youth sport programming within a reserve. The project was conceived on-reserve, with strategies that forefront practices relevant in Wikwemikong.

\section{CSP in Collective Cultures}

Despite the recognition among academics that CSP is a necessary and burgeoning line of inquiry, there are few examples where a cultural approach relevant to a marginalized population is considered in the sport and exercise psychology literature. From what little is known using CSP approaches, most examples are anecdotal and prescriptive. Among those featured, Kontos and BrelandNoble ${ }^{4}$ considered a number of populations, identified generally as people of color. Within their contribution, people of color referred to Americans from Asian, Arabic, Latino, and the North American Indian races. Pertaining to each population, the authors unpacked a few general guidelines by culture, upon which sport psychologists could search for within cultural nuances relevant to populations in their respective regions. On all counts, the said races shared a sense of family and community, belying their collective orientations. More specifically among the American Indians, it was acknowledged that the athletes regarded themselves in relation to their families, placing their family at the center, and their place in the family as a part of a larger whole. Affirming the views of cultural psychologists, ${ }^{14,15}$ athletes have been socialized along a continuum, with a highly individualistic orientation at one end, and a heavily weighted collective orientation at the other. ${ }^{2}$ Relevant to the present discussion is the focus on collective minded people, meaning those who ". . . value needs, wishes, and desires of groups over those of individuals and emphasize harmony, cooperation, cohesion, and conformity" (p. 7). ${ }^{2}$ When working with collective minded athletes such as those the academic researchers have encountered in Wikwemikong, it is now recognized that a catalyst to motivation is family and community, beyond strategies that affirm the athlete. It should be noted that the Wikwemikong members regard themselves as a collective, with practices that place family and community at the forefront, such as when an athlete represents Wikwemikong and many members from the community travel great distances to support that athlete. In our earlier work, we provided an anecdote from Wikwemikong's Sport and Recreation Director who encapsulated how Wikwemikong's members regard themselves as a collective: "In sport you're going to be wearing a uniform. Everybody looks the same, they're part of the team. They are wearing a uniform representing a larger community" (p. 407). ${ }^{8}$

Among those who catalyzed CSP, Hanrahan ${ }^{4}$ was among the first to realize that sport psychology services must account for cultural nuances. During a dance camp held for Australian Aborigine performing artists, it was recognized that her intended clients were seeking a bettersuited approach (to them) than the one most familiar to Hanrahan (ie, a mainstream European approach). When the author commenced her work in the context, she found that the best-suited approach encompassed strategies where the Aborigine culture predominated. Adapting to the demands posed during the bicultural exchanges, she considered the importance of process over outcome, the shared humility among the performing artists, and relevant to the present discussion, their sense of family and community as motivators. Hanrahan ${ }^{16}$ has recently expanded that, in relation to the same culture, social activities (eg, weddings or sporting events) are attended by a large number of family members. Family involvement also extends to family commitments among Australian Aborigines. For example, when a family demand is placed on Aborigine athletes, it is common for such athletes to place that demand over their own sport goals, thus foregoing training and even competition.

Just as family is prioritized among the aforementioned collective examples, there have been recent literary contributions where family among Canadian Aboriginal peoples has been overtly identified as a potent source of motivation, driving athletes to persist. Within these contributions, it has been found that an expansive web of family resources support aspiring Aboriginal athletes 
during training and competition. ${ }^{6}$ As part of an overall project developed to uncover the personal and social support strategies employed by 23 elite amateur and professional athletes, the participants shared the breadth of their family's involvement from sport entry onward through elite status. Emphasized were contributions not only from parents, but also from siblings, cousins, aunts and uncles, and grandparents. The roles of each member were not consistent across the athletes. For example, 1 athlete resourced her mother for inspirational stories applicable to sport. Others identified cousins and uncles as sport role models. Though no discrete roles could be assigned to any family member, there was a consistent message that many family members are involved in the development and success of Canadian Aboriginal sport performers, especially when those performers were born and raised on reserves. Consequently, when these researchers transitioned to a youth sport project to assist with Wikwemikong's health challenge posed by sedentary youth, a closer consideration of family support became a critical part of the project. ${ }^{8}$ Here, the role of family is considered in relation to the sport engagement of Canadian Aboriginal youth from Wikwemikong. Given the many Canadian Aboriginal peoples ${ }^{13}$ who have experienced a long history of oppression, the anecdotes and how they are presented reflects Aboriginal views and terminology through a series of de-colonizing methods that are facets of PAR. ${ }^{17}$ Within de-colonizing research the focus is on placing culturally relevant research methods (among the participants) at the forefront. In addition, what is achieved through the research in terms of lessons, strategies, affirmations of cultural identity, and expertise is prioritized.

\section{Methodology}

The methodology integrated 7 aspects: (a) the community, (b) the researcher team, (c) research assumptions, (d) the participants, (e) data collection, (f) analysis, and (g) trustworthiness.

\section{The Community}

As described in the introduction, Wikwemikong Unceded Indian Reserve is located in northern Ontario, Canada on the eastern side of Manitoulin Island. The reserve area totals 55,781 hectares and spans across Lake Huron and Georgian Bay. As the first permanent settlement on Manitoulin Island, Wikwemikong has retained much of its rich history and traditional culture. Wikwemikong is the largest of 7 Aboriginal communities on Manitoulin Island with an on-Reserve population of over 2700 people. The population consists of 3 different tribes, the Ojibway (Faith Keepers), Odawa (Traders), and Pottawatomi (Fire Keepers). The community is recognized as Canada's only Unceded Indian Reserve, meaning that its people have not relinquished title of their land to Canada's government. ${ }^{18}$

\section{The Research Team}

The Wikwemikong researchers hold a wide range of experiences and contributed their knowledge of sport practice and cultural sensitivity to this project. Researchers from Wikwemikong included the director of the sport and recreation program, members of his staff, a community sport coach, and the local high school principal. The Wikwemikong members contributed knowledge of the local school system and involvement in sport programming and cultural education. A university student researcher from Wikwemikong bridged the Aboriginal cultural community and academic university perspectives. Among the academic coresearchers were 3 Human Kinetics faculty members and 2 postgraduate students. The coresearch team held meetings once a month within the Aboriginal community during the project to discuss feedback for the development of questions, data collection procedures, data analysis, and verification. The community coresearchers verified coded data for accuracy in terminology and the development of themes. As well, the community meetings were integrated as a data source, ${ }^{8}$ reflecting the collective and participatory aspects of indigenous peoples. ${ }^{13,19}$ Further, the intent through the group discussions was to foreground the voices and decisions of the Aboriginal coresearchers throughout the project in a manner that is reflected forthcoming as participatory action research (PAR).$^{20}$ Reason has proposed that PAR be used as an approach to inquiry “. . . when the emphasis is on establishing liberating dialogue with oppressed people" (p. 262). ${ }^{20}$ Through a PAR approach, mainstream academic researchers from Northern Ontario, Canada have partnered with coresearchers from Wikwemikong Unceded Indian Reserve in the search for a solution to a community problem, namely the retention of Wikwemikong youth in sport and physical activity programming. As Patton has indicated in relation to PAR, it ". . . encourages joint collaboration within a mutually acceptable ethical framework to understand or solve organizational or community problems" (p.183). ${ }^{21}$

\section{Research Assumptions}

Several assumptions were inherent within the research project. First, when the Aboriginal coresearchers identified the topic for the present research initiative, they and the Wikwemikong Band and Council believed that the bicultural research team might uncover practical strategies that were not fully addressed by the community's sport and recreation staff and community youth center. As such, it was proposed that the community coresearchers approach the university researchers for support in the form of a formal research initiative. Second, the bicultural research team believed that intervention strategies must be multifaceted and targeted to various supportive resources in the Wikwemikong community in addition to youth participants. Third, it was assumed that the research team required culturally reflexive ways of asking questions, analyzing data, and presenting the 
findings meaningful to the participants. Consequently, it was believed that meaningful and sustainable solutions would result from the project. Fourth, the research team believed that mainstream programming strategies would be less relevant among the local Aboriginal culture than one developed in Wikwemikong.

\section{The Participants}

After the project was approved by the university's research ethics board as well as the Band and Council of Wikwemikong, community members from Wikwemikong were invited to engage in talking circles, and were contacted for initial circles through posted notices. During subsequent circles, the Wikwemikong Sport and Recreation staff and the local high school principal recruited participants, depending on which cohort was sought at the suggestion of the Wikwemikong coresearchers. All participants were identified by their role in relation to community youth sport participation, and they represented youth, family members, teachers, coaches, sport and recreation staff, and community elders. Initial talking circles (TC1-TC2) were held at the beginning of the project, open for all community members to participate and share their views related to youth sport programming strategies. Within the initial talking circles, 4 general questions were posed pertaining to sport barriers and possible solutions. Following community consultations, 6 age constrained talking circles, 2 for each cohort (TC3-TC8), were held in the community's high school to specifically seek input from youth by grade (ie, grades 7 to 8,9 to 10,11 to 12 ). The talking circles in the high school addressed emerging themes and areas from the data requiring further exploring, such as reasons for participating, or not participating, in sport. A final talking circle (TC9) was held for coaches and sport and recreation staff, addressing coaching barriers and programming expectations.

\section{Data Collection}

On reserves, talking circles are a traditional way of bringing people together with the intent of sharing knowledge. ${ }^{22}$ Talking circles provide a meaningful, respectful, and orderly social context for sharing views ongoing until there is nothing left to be said on the topic. ${ }^{23}$ Talking circles were employed in the current study as a culturally sensitive strategy somewhat akin to focus groups to elicit participants' thoughts and experiences regarding the role of families in support of youth involved in sport programming. It should be noted that in keeping with de-colonizing methodologies, ${ }^{13}$ research strategies must forefront the practices of the oppressed culture. The Wikwemikong coresearchers suggested the indigenous strategy of talking circles and believed that participants in Wikwemikong would be more comfortable sharing their experiences if the research practices were familiar to and affirming of them as a people. Each circle was led by a designated member of the community research team best suited to facilitate the sharing of knowledge. For example, if a talking circle was held with adolescents in the secondary school, the student representative on the project led. When circles included more adults than children they were led by senior community members of the research team (ie, an elder or the Sport and Recreation Director).

Talking circles consisted of 8 to 10 people and lasted approximately 2 hours. Participants were seated in a circular formation and asked one at a time to respond to a specific question or topic relating to youth sport programming within Wikwemikong. Initial talking circles were focused on family, community, and cultural aspects of youth sport (see Table 1). Subsequent talking circles were held in the high school to elicit further explanation of concepts discussed in earlier talking circles (see Table 2). For example, during the high school talking circles a student researcher from Wikwemikong led the talking circle and asked students to respond to the question, "What do grandparents do to support youth and keep them involved in sport and how do they do these things?" A stone was passed around the circle in a clockwise direction (in keeping with Wikwemikong tradition) with its possession indicating one's turn to speak, while the rest of the circle listened. ${ }^{22,23}$ Talking circles were concluded when all participants believed there was no more information to be added.

\section{Data Analysis}

Data were analyzed inductively based upon guidelines offered by previous researchers. ${ }^{17}$ First, talking circles were transcribed verbatim, followed by a thorough review of the documents (ie, misspelling, missing words). Second, provisional terms were assigned by the Wikwemikong student researcher and 2 graduate students throughout each talking circle transcript (TC1TC2) (eg, managing schedules, informal adoption). The categorization was considered to the point of consensus among the student researchers to develop and refine the thematic analysis. Third, provisional developments in categorization were presented to the Wikwemikong coresearchers during monthly meetings on reserve. During such meetings, the terminology was considered as well as the over-arching categorization of the data. Fourth, each emergent strategy was provisionally assigned by the first author and the student researchers to one (or several) family members (ie, support providers). The stakeholders included parents, aunts and uncles, siblings, cousins, grandparents, and the family as a whole. Fifth, the themes were presented as updates to the community coresearchers during subsequent monthly meetings, and refined through daylong discussions in Wikwemikong. Community involvement enhanced the relevance of coding, termed indigenous coding ${ }^{17,21}$ through the use of appropriate terminology and categorization. The community coresearchers considered the indigenous codes initially proposed by the Wikwemikong student, and among themselves, either confirmed the trends or regrouped the data until consensus was achieved. Each manuscript (3 in 


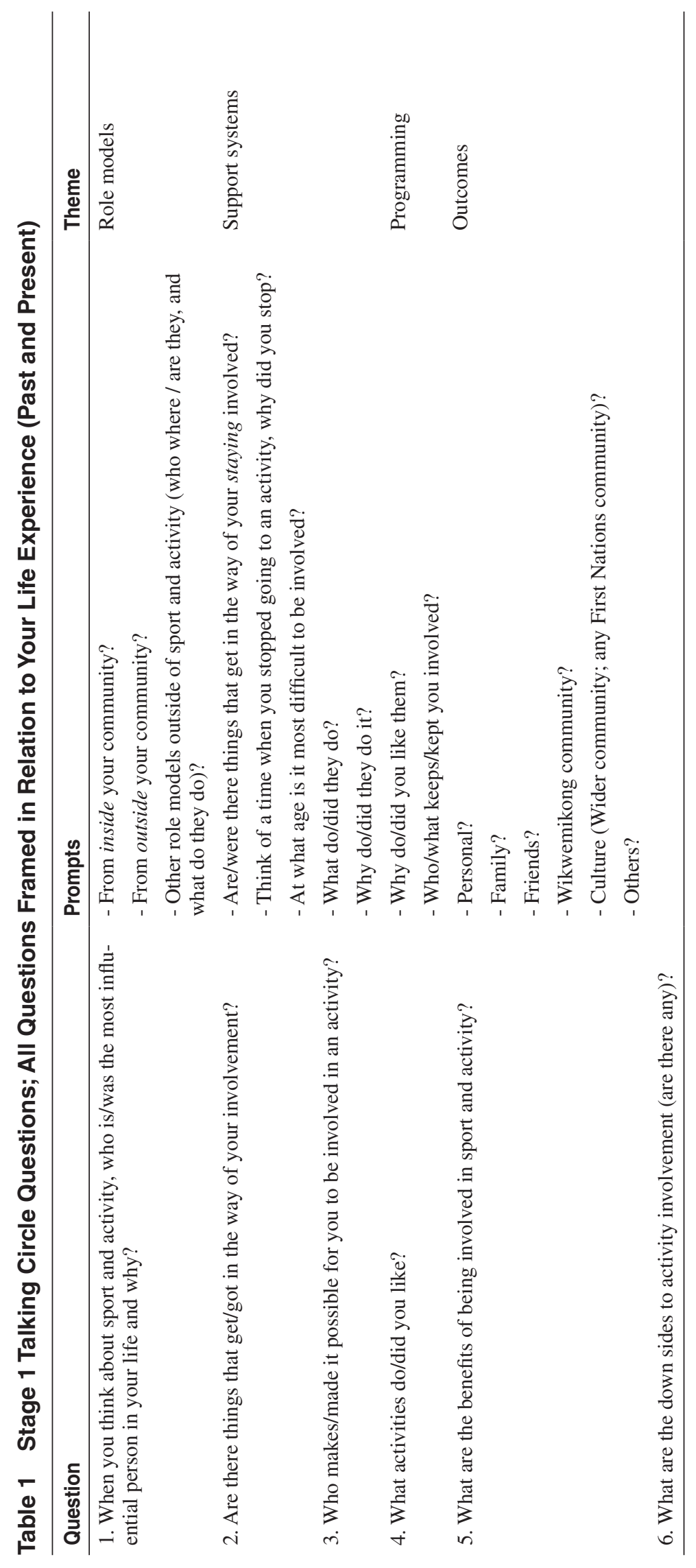


Table 2 Follow-up Questions With Youth Regarding the Role of Family in Sports; Preamble: The Aim With This Talking Circle Is to Gain a Better Understanding of Youth Sport Programs in Wikwemikong; The Information Gained Through This Talking Circle Will Provide Insight Into Who Helps You Attend Activities, as Well as the Barriers That Might Limit Your Participation; The Outcome of This Project Will Be the Development of Refined Youth Sport Programs Within Wikwemikong

\begin{tabular}{lc}
\hline Question & Prompts \\
\hline 1. What do grandparents do to support youth and keep them involved in sport? & - How do they do these things? \\
2. What do cousins do to support youth and keep them involved in sport? & - How do they do these things? \\
3. What do siblings do to support youth and keep them involved in sport? & - How do they do these things? \\
4. What do aunts/uncles do to support youth and keep them involved in sport? & - How do they do these things? \\
5. What does family in general do to support youth and keep them involved in sport? & - How do they do these things?
\end{tabular}

total pertaining to the role of community, athlete adaptation when off reserve, and the role of family) was coauthored by the academic and Wikwemikong coresearchers to enhance authenticity and forefront the involvement of the Wikwemikong coresearchers.

\section{Trustworthiness}

Three validity criteria ${ }^{24}$ aligned with the study, where the intent was to integrate aspects of the mainstream validity guidelines to ensure that peer-review guidelines would be met. In addition, transactional and transformational validity were integrated as part of the method, given the social implications of the project. Both types of validity follow below.

Maxwell's ${ }^{24}$ Guidelines. For descriptive validity, all talking circles were recorded using 2 audio recorders to avoid malfunction and missing data. The transcribers reviewed each recording before transcription, transcribed it verbatim, and then compared the transcript to the audio recording to enhance accuracy. The transcription phase was concluded with a research team member reviewing the transcripts for flow, completeness, and spelling. For interpretive validity, ongoing bicultural collaborations enhanced the depiction of Wikwemikong's standpoint. The Wikwemikong members provided contextual knowledge to facilitate the development of appropriate questions, proposed relevant research methods and an overarching PAR approach, assisted with the data collection-analysis process, and engaged in the coauthoring of publications and presentations. The theoretical validity of the current project was supported in 2 ways. First, the data were coded by 1 mainstream researcher and then refined with the help of a second mainstream member and the student researcher from Wikwemikong until consensus was achieved. All 3 members considered each piece of data and the white academic researchers deferred to the Aboriginal student regarding coding terminology. Second, the coded data were presented to community coresearchers to gather feedback on the importance of particular themes as well as to modify their categorization and labels. The data were presented through monthly updates, and within each meeting, time was scheduled to consider more closely matters of categorization and indigenous terminology. Precisely, the intent was to forefront the views of the Aboriginal coresearchers throughout the coding, thus supporting de-colonizing practices, all the while enhancing authenticity.

Cho and Trent's ${ }^{25}$ Guidelines. In qualitative research, transactional validity is an interactive process among the researcher(s), the researched, and the collected data, and the intent is to achieve consensus and accuracy. ${ }^{25}$ Within the current project various steps were developed to enhance transactional validity. First, talking circle questions were designed so that follow up questions could be asked to build mutual understanding among the 2 cultures. For example, when one participant attempted to describe the importance of family involvement in youth sport activities, one of the academic researchers followed up with an elaboration probe, and asked for more detail (he was not grasping what was being conveyed by the participant). In response the community participant asked for a pen and paper and subsequently drew what appeared to be a spider web. Within it she labeled different family members, and in so doing conveyed the integrated involvement of family when it comes to youth sport engagement. Second, at each stage of data analysis, community coresearchers were consulted to verify that interpretation was congruent with the values and beliefs of Wikwemikong. A university student from Wikwemikong participated to assist with data analysis so that Aboriginal cultural values would be prevalent throughout the entire research process.

Transformational validity was also pursued throughout the study. The data informed the development of leadership training programs for youth and the sport staff. As a part of the implementation process community members have already assumed responsibility for the program, manifesting in self-governance by Wikwemikong. Presently, the programming is in its second year, with commitments on the part of the community to extend the programming for 3 additional years. Further, the Wikwemikong coresearchers have 
led dissemination efforts for both cultures. Dissemination in the Aboriginal community has been at the local level through community meetings, youth presentations within the schools, and within North America through presentations at 2 successive North American Indigenous Games conferences. Among the academic audience, the bicultural research team has presented over 4 successive years at international conferences, and they have also submitted manuscripts to peer reviewed outlets. The ongoing research has been instrumental in establishing trusting bicultural relationships, and the members are presently planning their next research / practical initiative.

\section{Results}

From the project database of 933 anecdotes, 241 belonged in the boundaries of family support of youth in relation to sport programming, accounting for $25.8 \%$ of the data. Additional data outside the parameters of the present submission pertained to community strategies and the sport participants' personal strategies. From the data pertaining to family support, the following strategies were identified: (a) determining direction, (b) managing priorities, (c) finances, (d) transportation, (e) support and encouragement, (f) role models, and (g) mentoring (see Table 3. Family identified as offering the aforementioned strategies included: parents, aunts and uncles, siblings, cousins, grandparents, and family in general (referring in totality to all family members previously mentioned). Sample anecdotes selected by consensus among the community coresearchers through monthly meetings exemplify themes. Each anecdote, comprised of a response from a Wikwemikong member (either coresearcher or participant) was coded according to whether it came from a talking circle (TC) or community meeting (M) followed by a number indicating each sequential data collection (eg, M3 = third community meeting).

\section{Determining Direction}

The initial decision to be involved in a sport can be a difficult process. There are many decisions to make about joining a sports league including availability, cost, location, and motivation to participate. During the talking circles the community members identified parents as having a role in decision making and determining a child's initial direction in sport. "It's like parenting, you have to look after your kids, and part of it is involving them in things and setting schedules." (M3) The interests of the household where the child grows up plays an important role in what activities they pursue as they become older. While some parents encourage their kids to participate in sports there are others that discourage participation: "Some parents are pressuring the kids to quit sport and physical activity either because it is too expensive or takes up too much time." (M4)

\section{Managing Schedules}

One of the challenges parents and youth mentioned that interferes with the ability to participate in activities was managing schedules. "Everybody in the family will have different things they have to do." (M3) Managing multiple schedules involved identifying individuals' priorities and relying on family members to help children attend activities. One resource that parents sought out was assistance from grandparents:

Like tomorrow, there's a tournament [grandchild] is playing in. The little one's playing in Gore Bay, [one grandchild] has to be in Sudbury and [another grandchild] has to be somewhere. So there's one extra body needed to go to Sudbury, that's me. (M3)

Youth frequently mentioned having to choose between activities when 2 were scheduled at the same time, often resulting in the youth giving up an activity. "I stopped figure-skating because I had hockey and soccer." (TC3) At the same circle, a second participant affirmed: "If I

Table 3 Anecdotes ${ }^{a}$ Related to Family Support of Youth in Sport Programming

\begin{tabular}{|c|c|c|c|c|c|c|c|}
\hline Who/what & Parents & $\begin{array}{l}\text { Aunts/ } \\
\text { uncles }\end{array}$ & Siblings & Cousins & $\begin{array}{l}\text { Grand- } \\
\text { parents }\end{array}$ & $\begin{array}{l}\text { Family in } \\
\text { general }\end{array}$ & Total \\
\hline Determining direction & 17 & 0 & 0 & 0 & 5 & 0 & 22 \\
\hline Managing schedules & 11 & 0 & 0 & 0 & 0 & 0 & 11 \\
\hline Financial concerns & 13 & 1 & 0 & 0 & 7 & 0 & 21 \\
\hline Transportation concerns & 8 & 2 & 0 & 1 & 7 & 1 & 19 \\
\hline Encouragement and commitment & 29 & 11 & 4 & 7 & 10 & 13 & 74 \\
\hline Role models & 25 & 4 & 8 & 5 & 5 & 4 & 51 \\
\hline Mentoring & 0 & 5 & 3 & 7 & 1 & 0 & 16 \\
\hline Other & 0 & 0 & 1 & 1 & 2 & 23 & 27 \\
\hline Total & 103 & 23 & 16 & 21 & 37 & 41 & 241 \\
\hline
\end{tabular}

${ }^{a}$ Each cell represents the number of anecdotes in each category. 
have a different sport at the same time. This year I had to stop playing rep. (a community designated athlete) lacrosse because the provincials is on the same time as my hockey try-outs." (TC3)

\section{Finances}

During the talking circles community members were asked what barriers youth face when participating in sport, specifically, what prevents them from being able to join a team or continue playing with a team. "They don't have the means to stay in the sport, the money or the support." (M3) The decision to participate in a sport frequently came down to having the funds to join a league and then purchase equipment ( 2 financial challenges). When speaking with the youth directly, they were asked who pays for their involvement. Parents were referenced for providing financial support to participate. "My dad. He takes the time and money to put me into sports because he likes to keep me active." (TC3) There are also associated costs including equipment and fundraising for uniforms and tournaments. Supplementing support is often received from grandparents as well as aunts and uncles. "My grandma really helped me with fundraising for hockey tournaments and she was at my hockey games." (TC5) Further, "When they see an ad for sports like say soccer camp, my grandparents and aunts and uncles will say I should join. They'll help get me started, buying me soccer shoes." (TC5)

\section{Transportation}

Community members identified the availability of transportation as a deciding factor influencing youth sport participation. Some community members placed the responsibility of transportation on parents. "The parent has to be dedicated to make sure to get all the transportation organized." (M3) Especially coaches identified transportation as a necessity for keeping youth in sports.

I have my practice at the ballpark for track and field. I said it's your responsibility to have your own ride home. It's the parents with the support to pick up their kids. I drop them off at the Band office. If they get rides from there, that's a start. (TC7)

Youth acknowledged the importance of having reliable transportation to attend practices and games. "My mom takes me to my games and my practices, and drives me back and forth. She gets me ... she makes sure that I stay in what I'm doing and she makes sure I keep up with the team." (TC3) When parents were unable to provide transportation community members identified grandparents as the individuals most likely to provide transport. "I play hockey, when my mom and dad didn't have enough gas in the car to take me to the games. My grandma would drive me." (TC6) In addition to grandparents, youth also identified aunts and uncles as sources of transportation. The need for reliable transport increased as youth advanced to higher levels of competition in their sport and thus relied on more family resources for transportation.

\section{Support and Encouragement}

Youth often referred to the importance of their family members providing support and encouragement for sport activities. Beyond what has already been exemplified pertaining to parents and grandparents, one youth indicated that her sister encouraged her to be active. "My sister always tries to get me to go do stuff with her that involves activities out of doors. I try to do that as much as possible with her." (TC6) Other youth indicated that their siblings and cousins were sources of motivation. "They encourage me and I got to play against some of my cousins so it made it a lot more competitive. They told me not to quit." (TC5) As discussions broadened, being active as a family and playing sports together were important when encouraging youth to lead an active lifestyle. One youth indicated he enjoyed playing "hockey, volleyball, soccer because I play with my family." (TC4) Finally, support also has a positive motivational impact on performance when competing in sport. Youth felt that they were more likely to try their best when they had family present at games, speaking to the importance of the collective.

I have younger cousins playing hockey in Sudbury and every game they've got uncles and aunts that watch their games. I guess they're accountable to themselves that they play hard every game knowing that there's someone watching every game. (M2)

\section{Role Models}

When youth were asked about why and how they had made choices to participate in sport they indicated it was what they had observed while growing up. "It was role modeling. You had somebody showing you. Maybe not formally instructing you, but they were your example." (M2) Youth that observed family members participating in sports indicated that they were more likely to be active and involved in those same sports. "My mother was a runner and so was my aunt and I watched them skate and I watched them run, play ball when I was growing up." (M2) Although youth indicated various family members as role models only grandparents acknowledged their role as a role model. "I've always carried it on, I don't know how many other grandparents can say that. Because I myself exercise.” (M2)

\section{Mentoring}

Contrasting with role models, mentors are aware of their relationship with the person they are mentoring. Mentoring was seen as a "hands on" approach where teaching and showing are emphasized. Most frequently siblings were regarded as important mentors for learning and developing sports skills. As playmates, older siblings handed down skills to younger siblings.

Mine would probably be my brother because he taught me how to play different sports I wanted to play. He always spent time with me when I was younger and taught me how to play. He played with me constantly. So he was my role model. (TC4) 
Older siblings often looked to aunts and uncles to learn new sports and skills. When asked who taught youth to play sports, one youth responded "My uncle because he's the one that taught me all the sports." (TC3) When youth were seeking sport advice they often asked their grandparents. "They [grandparents] have been through it all; most of it all anyway. They're not done going through it all, but I learned from them all the time, answering questions." (TC2)

\section{Discussion}

The purpose with the current study was to delineate the supportive strategies provided by Aboriginal families in one reserve (Wikwemikong) for youth involved in its sport programs. Family support was inclusive of parents, grandparents, aunts and uncles, siblings, and cousins. Consistent with previous findings, ${ }^{6}$ the current study clarifies sources (ie, family members) and indigenous types of support relevant to Canadian Aboriginal youth. Paralleling the aforementioned work, the role of family in a youth's sport experience resembles a web of interrelated resources, where supportive strategies are often assigned to many family members depending on time demands and financial circumstances. For example, the Wikwemikong participants and coresearchers clarified that physically active parents encouraged their children to persist in sport and activity. However, it was also found that grandparents, and even aunts and uncles would drive the youth to their activities. The emphasis with the current study was on supporting the Wikwemikong in the creation of a community-refined initiative for its youth (family membership structured within), founded upon the views of its own membership. Even with a regional focus (reflecting a community proposed initiative), it was found that breadth of family involvement affirms the collective nature of Aboriginal peoples. ${ }^{4,7,23}$ A community elder involved as a coresearcher in the project explained to our group that it takes a community to support a sport participant. What the present work contributes is a practical explanation not only of why mainstream sport initiatives with an emphasis on the sport participant mostly, might fall short in collectively minded Aboriginal communities such as Wikwemikong. There is also an explanation of how a collective culture works together, in this case through its collectively minded families, to enable youth participants to embark early on in sport and activity. Activity involvement is not easy when families reside in economically challenged communities, and where there are abundant health risks posed from youth, onward. ${ }^{9-12}$ Consequently, the approach to understanding and subsequently designing effective sport programs within such communities requires a broad range of family members, all assisting with the logistics and financial demands posed by continued enrollment. Through an appreciation of family resources, and how these might work collaboratively, sport and physical activity staff can appreciate the potential challenges encountered by Aboriginal youth in sport, and perhaps assist in problem solving techniques that reaffirm the expansive resources that can foster adherence.

Beyond what was gleaned from the content presented in this study, there are also considerations pertaining to the research methodology. During an earlier project, our coauthors sought the voices of elite amateur and professional Aboriginal athletes, in terms of their personal and social support strategies. From what was learned, it became clear to the researchers that traditional Aboriginal sport participants employ motivational practices that differ from what has often been published in relation to mainstream participants (eg, the use of elders in place of sport and exercise psychologists ${ }^{7}$ ). However, our bicultural research team has come to realize that more pressing than the proliferation of such research is the spirit in which the research is done. ${ }^{17}$ There is a distinction between research about cultures, and culturally reflexive research. ${ }^{13,19}$ The former encompasses mainstream methods employed to garner data from the cultural other though mainstream techniques. What we have attempted here is to integrate methodologies indigenous among the Wikwemikong in a manner that forefronts and empowers. For example, the project (and this specific study) was proposed by the Wikwemikong, with strategies that will be integrated in the said community for its youth through a self-governed initiative led by sport staff and youth leaders. In addition, community discussions and talking circles were employed in place of interviews and focus groups. Finally, the present research reflects the voices of Wikwemikong appointed coresearchers who were active throughout the research.

One potential limitation in applying the knowledge that has been derived from the current project is that Wikwemikong members believe in noninterference, even when working with others in their community. ${ }^{26}$ Noninterference is a philosophy where each person be allowed to make the necessary mistakes through firsthand experience. On a positive note, this philosophy has allowed the academic researchers to enter into a research project in collaboration with Wikwemikong, and to learn through lived experience, missteps, and readjusted approaches. However, our team now proposes that what is achieved through research, both in terms of the project focus and how community coresearchers are fore-fronted, can (and should) become an integral part of our cultural research, extending beyond matters of methodological techniques alone.

There are opportunities and also challenges posed through bicultural research projects such as ours. The opportunities should academic researchers in the mainstream be able to engage people from an oppressed cultural community are several. First, there is an opportunity to work with an oppressed people, where research can serve to forefront a standpoint that might have been positioned at the margins, and in so doing, encourage silenced voices to speak once again. Second, with new perspectives might come novel and effective strategies to problems that transcend one culture such as youth inactivity, and potentially, longer term health risks caused by sedentary lifestyle. Research methodologies are culturally 
informed. There are a vast number of possible research methods available to researcher in human kinetics, should we invite a closer integration of culture into our work. Beyond the more general benefits aforementioned, the academic researchers have learned a considerable amount of how we need to go about doing our work. There now is a push to engage in research where we feel our work makes a difference for someone. Despite all of the positive aspects we have outlined, we have also encountered challenges over our funded projects. For example, there is sometimes an obligation to do many things beyond a perfunctory project through research among a mainstream and an oppressed culture. However, the challenge of restorative/de-colonizing projects is that they are set to time lines, especially when the commitments are tied to funding. Such time lines are relatively short in relation to the years of amassed oppression, and what is gained in research benefits are often promising as an implication, and they are not immediately evident. Compounding the challenge to do what is correct through the research, the promise of improvement is offset by distrust that has been long-standing. Consequently, those academics who choose to engage in research with oppressed people need to be mindful that commitments are extensive in and through time. An additional challenge is that with de-colonizing methods, the academic researchers must do themselves out of their job, and encourage those within the oppressed culture to step forward and take ownership of what is derived through the project. In so doing, there is an adjustment for both cultures as one group steps forward and the other steps back. Typically, there is no such concern as researchers have a proprietary ownership over their work and what is derived from that work. Despite the complexities associated in PAR research such as ours, the authors from both cultures believe that the efforts in and through the research far outweigh any challenges.

\section{Conclusions and Implications}

Ongoing collaborative efforts among the coresearchers have been instrumental in continuing the research project and moving toward practical implementation. From the data, community appropriate scenarios and case studies have been included in youth programs to reflect the important role of families in leadership development. Within the leadership program youth are encouraged to resource family support in relation to the aforementioned family strategies. The web structure does not place a hierarchy to support; rather it emphasizes the importance of every person's contribution as part of a collective. Providing leadership training allows individuals to complete the circle of sport programming, by beginning as a sport participant in their youth, and later passing those sport skills on to the next generation. The first graduates have begun implementing their skills by running summer activities in Wikwemikong for youth. Consequently, the current study has been energized by Wikwemikong's members taking on roles in the project, while also contributing to youth sport programs.
From this project we recommend that future CSP researchers use community relevant strategies in addition to culturally relevant strategies when developing research projects. Quality involvement can be achieved by actively involving community members from the intended culture in the project from development, onward. Further, when the intended culture is an oppressed culture, we propose that consensus building models be employed throughout the process, and that what is learned be developed into a community relevant initiative governed by the community itself. These recommendations contribute to the advancement of social change and facilitate improved relations among cultures.

\section{References}

1. Ryba TV, Wright HK. From mental game to cultural praxis: a cultural studies model's implications for the future of sport psychology. Quest. 2005;57:192-212.

2. Schinke RJ, Hanrahan SJ, Catina P. Introduction to cultural sport psychology. In: Schinke RJ, Hanrahan SJ, eds. Cultural Sport Psychology. Champaign, IL: Human Kinetics; 2009:3-11.

3. Kontos AP, Arguello E. Sport psychology consulting with Latin American athletes. Athl Insight. 2005 [cited 2008 May 10]. Available from www.athleticinsight.com/ Vol7Iss3/LatinAmerican.htm

4. Kontos AP, Breland-Noble AM. Racial/ethnic diversity in applied sport psychology: a multicultural introduction to working with athletes of colour. T Sport Psychol. 2002;16:296-315.

5. Hanrahan SJ. Sport psychology and indigenous performing arts. T Sport Psychol. 2004;18:60-74.

6. Schinke RJ, Eys MA, Danielson R, et al. Cultural social support for Canadian aboriginal elite athletes during their sport development. Int J Sport Psychol. 2006;37:330-348.

7. Schinke RJ, Hanrahan SJ, Eys MA, et al. The development of cross-cultural relations with a Canadian Aboriginal community through sport psychology research. Quest. 2008;60:357-369.

8. Blodgett AT, Schinke RJ, Fisher LA, et al. From practice to praxis: community-based strategies for Aboriginal youth sport. J Sport Soc Issues. 2008;32:393-414.

9. Health Canada. Aboriginal diabetes initiative [report on the internet]. 2004 [cited 2007 Mar 22]. Available from www.hc-sc.gc.ca/fnihb/cp/adi/introduction.htm.

10. Adrian M, Payne N, Williams RT. Estimating the effects of Native Indian population on county alcohol consumption: the example of Ontario. Int J Addict. 1991;2:731-765.

11. Scott K (Kisht Anaquot Health Research and Program Development). Indigenous Canadians: Substance Abuse Profile 1995. National Native Alcohol and Drug Abuse Program; 1995.

12. Health Council of Canada. Aboriginal Health 2006 Annual Report [report on the internet]. 2007 [cited 2008 Jun 10]. Available from www.healthcouncilcanada.ca.

13. Smith LT. Decolonizing Methodologies: Research and Indigenous Peoples. Dunedin: University of Otago Press; 1999.

14. Norenzayan A, Nisbett RE. Culture and causal cognition. In: Ruscher JR, Hammer EY, eds. Current Directions in Social Psychology. Upper Saddle River, NJ: Pearson; 2004:55-60. 
15. Williams JE, Satterwhite RC, Saiz JL. The Importance of Psychological Traits: A Cross-Cultural Study. New York: Plenum Series in Social/Clinical Psychology; 1998.

16. Hanrahan SJ. Working with Australian Aboriginal athletes. In: Schinke RJ, Hanrahan SJ, eds. Cultural Sport Psychology. Champaign, IL: Human Kinetics; 2009:185-192.

17. Schinke RJ, Peltier D, Hanrahan SJ, et al. The progressive integration of mainstream research practices and Canadian Indigenous culture. Int J Sport Exerc Psychol. 2009; in press.

18. Manitoulin Island Website. 2005 [cited 2008 Jul 19]. Available from www.manitoulin-island.com/wikwemikong/.

19. Canadian Institute of Health Research. CIHR Guidelines for Health Research Involving Aboriginal People. Ottawa, Canada: Author; 2007.

20. Reason P. The approaches to participatory inquiry. In: Denzin NK, Lincoln YS, eds. Strategies for Qualitative Inquiry. Thousand Oaks: Sage; 1998:262-291.
21. Patton MQ. Qualitative Research and Evaluation Methods. 4th ed. Thousand Oakes, CA: Sage; 2002.

22. Running Wolf P, Rickard JA. Talking circles: a Native American approach to experiential learning. J Multicult Couns Devel. 2003;31:39-43.

23. Picou JS. The "talking circle" as sociological practice: cultural transformation of chronic disaster impacts. Socio Pract J Clin Appl Sociol. 2000;2:77-97.

24. Maxwell JA. Understanding and validity in qualitative research. In: Huberman AM, Miles MB, eds. The Qualitative Researcher's Companion. Thousand Oaks, CA: Sage Publications; 2002:37-63.

25. Cho J, Trent A. Validity in qualitative research revisited. Qual Res. 2006;6:319-340.

26. Martin-Hill D, Soucy D (Indigenous Health Research Development Program). Ethical guidelines for Aboriginal research elders and healers roundtable. Report. The Interagency Advisory Panel of Research Ethics. 